\title{
Initiation And Persistence With Antiplatelet Agents Among The Patients With Acute Coronary Syndromes: A Retrospective, Observational Database Study In China
}

This article was published in the following Dove Press journal:

Patient Preference and Adherence

\author{
Xin Liu' \\ Xiaoning $\mathrm{He}^{\mathrm{I}}$ \\ Jing $\mathrm{Wu}^{\mathrm{I}}$ \\ Da Luo',2
}

'School of Pharmaceutical Science and Technology, Tianjin University, Tianjin, People's Republic of China; ${ }^{2}$ Tianjin Health Development Research Center, Tianjin, People's Republic of China
Correspondence: Jing Wu

School of Pharmaceutical Science and

Technology, Tianjin University, 92 Weijin

Road, Nankai District, Tianjin 300072,

People's Republic of China

Tel +862227405176

Fax +862287401830

Email jingwu@tju.edu.cn

Da Luo

School of Pharmaceutical Science and

Technology, Tianjin University, Tianjin,

People's Republic of China

Email vividvigorous@163.com
Objectives: To investigate the initiation and persistence of antiplatelet agents and the associated factors for patients with acute coronary syndromes (ACS) in Tianjin, China.

Methods: Data were obtained from Tianjin Urban Employee Basic Medical Insurance database (2011-2015). Adult patients who were discharged alive after the first ACS-related hospitalization (index hospitalization) between January 2012 and December 2014 were included. Patients who initiated with antiplatelet therapy, including aspirin monotherapy, clopidogrel monotherapy, or dual antiplatelet with aspirin and clopidogrel at discharge or within the initial 30-day follow-up after discharge were further identified. Patients with no gaps of $\geq 30$ days in antiplatelet therapy were deemed persistent. The logistic model and Cox model were used to explore the associated factors of initiation and persistence with antiplatelet agents, respectively.

Results: In total, 21,450 patients $(64.6 \pm 10.7$ years; $46.0 \%$ female $)$ were included. Only $70.3 \%(\mathrm{~N}=15,071)$ of them initiated with antiplatelet agents within the initial 30-day followup; $85.0 \%(\mathrm{~N}=12,809)$ of the initial users discontinued their antiplatelet therapy, and the average time to discontinuation was $117.4 \pm 119.7$ days. The patients who had prior antiplatelet agents utilization (Odds ratio $[95 \% \mathrm{CI}]=1.93[1.78-2.09]$; hazard ratio $[95 \% \mathrm{CI}]=0.78$ [0.74-0.81]), received percutaneous coronary intervention (PCI) during the baseline period $(\mathrm{OR}=1.47$ [1.26-1.73]; HR=0.91 [0.84-0.97]) or index hospitalization $(\mathrm{OR}=22.40$ [18.6326.92]; $\mathrm{HR}=0.51$ [0.49-0.53]) were more likely to initiate and persist with antiplatelet agents, while the female $(\mathrm{OR}=0.75$ [0.70-0.81]; $\mathrm{HR}=1.22$ [1.88-1.27]) patients were less likely to initiate and persist with antiplatelet agents.

Conclusion: The initiation and persistence with antiplatelet agents are poor among the ACS patients in Tianjin. Females are associated with poorer initiation and persistence, while prior antiplatelet agents use and receiving PCI during baseline period or index hospitalization are associated with better initial use and better persistence.

Keywords: acute coronary syndromes, antiplatelet agents, initiation, persistence, China

\section{Introduction}

Acute coronary syndrome (ACS), including unstable angina (UA), non-ST segment elevation myocardial infarction (NSTE-MI), and ST segment elevation myocardial infarction (STE-MI), is an umbrella term for situations where the blood supplied to the heart muscle is suddenly blocked..$^{1-3}$ Rupture of coronary atherosclerotic plaque, vasospasm, and consequent platelet adhesion, aggregation and secondary 
thrombosis were the major causes of ACS. With the specific pathological base, the ACS patients face long-term high risk of recurrence and other potential adverse cardiovascular events, even death during secondary prevention. ${ }^{4-8}$ This disease seriously affects the quality and length of life. ${ }^{3,9}$ As one of the most widespread causes of morbidity and mortality, ACS not only threatens human health seriously but also presents a significant burden for healthcare providers globally. ${ }^{10-12}$

Preventing platelet activation and subsequent aggregation are the key targets in the management of ACS. ${ }^{13}$ Guidelines of the American College of Cardiology/ American Heart Association (ACC/AHA), European Society of Cardiology (ESC), and China Heart Society (CHS) all recommend antiplatelet therapy use for ACS patients in the acute setting. ${ }^{14}$ For all ACS patients without contraindications, aspirin is recommended for long-term use and a $\mathrm{P}_{2} \mathrm{Y}_{12}$ inhibitor, eg, clopidogrel, prasugrel, or ticagrelor, is recommended for up to 12 months. ${ }^{15}$ As the first $\mathrm{P} 2 \mathrm{Y}_{12}$ inhibitor which entered the Chinese market, Clopidogrel is the most widely used $\mathrm{P}_{2} \mathrm{Y}_{12}$ inhibitor which is recommended by Chinese diagnostic and therapeutic guideline for ACS, ${ }^{9,16}$ and is also the only $\mathrm{P}_{2} \mathrm{Y}_{12}$ inhibitor reimbursed by the basic medical insurance system in China during the study period.

Many studies suggested that optimal antiplatelet therapy according to the guidelines will decrease the risk of adverse cardiovascular events, such as stroke, myocardial infarction (MI), and all-cause mortality. ${ }^{4,17,18}$ However, many patients do not initiate with antiplatelet agents after ACS or cannot persist to the regimen prescribed to them. ${ }^{19,20}$ The prior studies suggested that $10-20 \%$ of ACS patients were left untreated within the initial period after discharge. ${ }^{21,22}$ It was also reported that, after 12-month follow-up, about half of the patients did not persist to use antiplatelet agents. ${ }^{18,23} \mathrm{It}$ is evident that there is room for improvement in the prevention management of ACS. Despite some related studies being reported abroad, ${ }^{24-26}$ corresponding observational studies using real-world data are lacking among the Chinese population. This study aimed to evaluate the initiation and persistence of antiplatelet agents and explore their associated factors among patients with ACS in Tianjin, China.

\section{Methods}

\section{Data Source}

The data in this retrospective study were extracted from Tianjin Urban Employee Basic Medical Insurance (UEBMI) from January 1, 2011 to December 31, 2015. As the most extensive basic medical insurance system in China, the UEBMI system covers all employed workers and retirees from both public and private sectors in Tianjin. By 2015, the UEBMI covered almost 5.2 million unique members in Tianjin, representing $50.8 \%$ of the registered residents. ${ }^{27}$ The analytical sample in this study was a $30 \%$ random sample of all enrollees. The dataset contained the unique patient-level demographic characteristics, pharmacy claims (quantity, strength, date of prescription), medical claims of inpatient and outpatient services, and related medical and medication costs of employees and retirees in Tianjin. The Tolerability and Ethics Committee at the School of Pharmaceutical Science and Technology, Tianjin University, waived the requirement of ethics approval for the current study, as this was a retrospectively observational study using the de-identified medical claims data.

\section{Study Population}

Adult patients who were discharged alive with primary discharge diagnosis of ACS (ICD-10 code I20.0, I21, I22 supplemented by Chinese description) from January 1, 2012 through December 31, 2014 were identified. The first qualified hospitalization during the identification period was defined as the index hospitalization. Patients with malignant diseases, who had no continuous insurance enrollment for 12 months before (baseline period) and 12 months after (follow-up period) the index hospitalization, or who experienced major adverse cardiovascular events (MACEs) which included MI, stroke, and death within the initial 30 days after discharge were further excluded. The included patients were followed up from the discharge of index hospitalization until the first MACE or 12 months after this discharge, whichever came first.

\section{Outcome Measures}

\section{Baseline Characteristics}

Baseline information was reported, including demographic characteristics (age, gender, and employment status), clinical characteristics (Charlson Comorbidity Index [CCI)], medical history during baseline), prior medication utilization (antiplatelet agents, angiotensin-converting enzyme inhibitors/ angiotensin receptor blockers [ACEIs/ARBs], $\beta$-blockers, statins and calcium channel blockers [CCB]), prior procedure received (percutaneous coronary intervention, PCI) and health care resource utilization (HRU) and direct medical costs. The procedure received (PCI or not) and the length 
of stay (LOS) during the index hospitalization were also described.

\section{Initiation}

Initial use of antiplatelet treatment was examined by the presence of the first claimed antiplatelet prescriptions at discharge or within the initial 30 days after the index discharge. Specifically, patients who had at least one prescription of aspirin or clopidogrel during the timeframe mentioned above were defined as the initial users.

\section{Persistence}

Persistence was analyzed among the initial users. Patients who had no gap of $\geq 30$ days between the timepoint of exhausting the supplied antiplatelet agents and the following refill during the follow-up period were defined as persistent. Persistence was measured by the rate of discontinuation and persistence duration, ie, time (days) to discontinuation, which was calculated as the number of days between the first day of the initial prescription and the last day of the last prescription before the discontinuation. Overlaps of the prescriptions were not accumulated.

Clopidogrel was the only drug of $\mathrm{P}_{2} \mathrm{Y}_{12}$ that can be reimbursed by UEBMI during the study period. Based on the recommend guidelines and the reimbursement policies, the antiplatelet agents in this study mainly consist of aspirin and clopidogrel.

\section{Statistical Analysis}

The continuous variables were reported as mean and standard deviation (SD), and the categorical variables were described using frequencies and percentages. Differences between two subcohorts were assessed using Chi-squared tests for categorical variables, and $t$-tests for continuous variables. Logistic regression model was used to explore the potential factors associated with initiation of antiplatelet agents, and Cox's proportion hazard model was used to explore the associated factors for persistence. Demographics (age, gender, and employment status), clinical characteristics (CCI, medical history, prior medication utilization, and PCI received), baseline HRU and direct medical costs (any hospitalization, any outpatient visit, total direct medical cost, and LOS of index hospitalization) were included as potential associated factors in the regression models. A two-side P-value of $<0.05$ was considered statistically significant.

All statistics analyses were conducted using STATA version 12.1 .

\section{Results}

\section{Patient Characteristics}

Figure 1 shows the patients flow chart with the number of patients included and excluded in the study. A total of 25,397 adult patients who had a primary discharge diagnosis of ACS were identified between 2012 and 2014, among which 21,450 eligible patients were included as the study population.

In Table 1 , the mean (SD) age of the study population was 64.6 (10.7) years, $46.0 \%$ were female and $80.5 \%$ were retired. The mean (SD) CCI was 1.7 (1.6). The most common co-morbidities during the baseline period among ACS patients were hypertension (75.0\%), hyperlipidemia $(46.6 \%)$, and diabetes (37.5\%). Among these patients, $54.7 \%(\mathrm{~N}=11,728)$ had prior use of an antiplatelet prescription during the baseline period, $31.1 \%$ patients experienced $\geq 1$ all-cause hospitalizations during the baseline, and the average annual number of outpatient visits was 30.5 (31.7) among the patients. The mean direct medical cost of the patients was $12,772(18,893) \mathrm{CNY}$. The LOS was 10.5 (5.5) days on average and 36.5\% of patients received PCI during the index hospitalization.

\section{Initiation With Antiplatelet Treatment And Associated Factors}

About $70.3 \%(\mathrm{~N}=15,071)$ of the total patients had the initial antiplatelet prescription within 30 days after discharge, while $29.7 \%(\mathrm{~N}=6,379)$ of patients did not have any antiplatelet agents. The percentages of initiation with aspirin or clopidogrel were similar, which were $47.4 \%$ $(\mathrm{N}=10,160)$ and $47.7 \%(\mathrm{~N}=10,226)$, respectively, among ACS patients during the initial 30 days (Table 2).

The initial $(\mathrm{N}=15,071)$ and non-initial $(\mathrm{N}=6,379)$ patient subcohorts differed on most of the baseline characteristics. The result suggested that age, gender, medical history, prior use, baseline MRU and index characteristics were all associated with initiation (Table 3). Compared with patients aged $\leq 54$, patients aged older were more likely to initiate antiplatelet treatment (age 55-64: adjusted Odd Ratio $[95 \% \mathrm{CI}]=1.46 \quad[1.31-1.63]$; age $65-74$ : adjusted $\mathrm{OR}=1.82 \quad[1.61-2.07] ;$ age $\geq 75$ : adjusted $\mathrm{OR}=1.70$ [1.49-1.93], respectively). The patients who had higher CCI (adjusted OR=1.04 [1.00-1.07]) had prior MI (adjusted OR=1.18 [1.07-1.30]) or had baseline antiplatelet prescription (adjusted $\mathrm{OR}=1.93$ [1.78-2.09]) were more likely to initiate with antiplatelet treatment. Also, receiving PCI during the baseline period (adjusted 
Insured who enrolled in Urban Employee Basic Medical

Insurance (UEBMI) during 2012-2014

$$
\mathrm{N}_{1}=6,996,844
$$

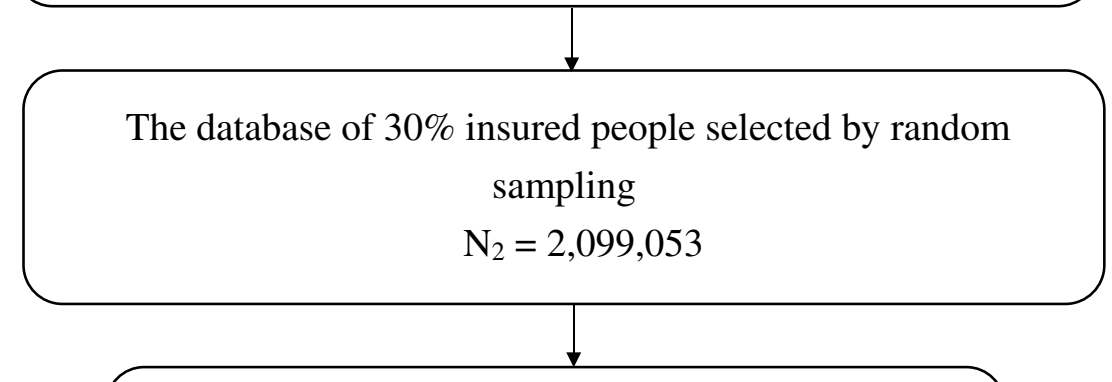

Had $\geq 1$ primary discharge diagnosis of ACS during 2012.1.1-2014.12.31

$$
\mathrm{N}_{3}=25,397
$$

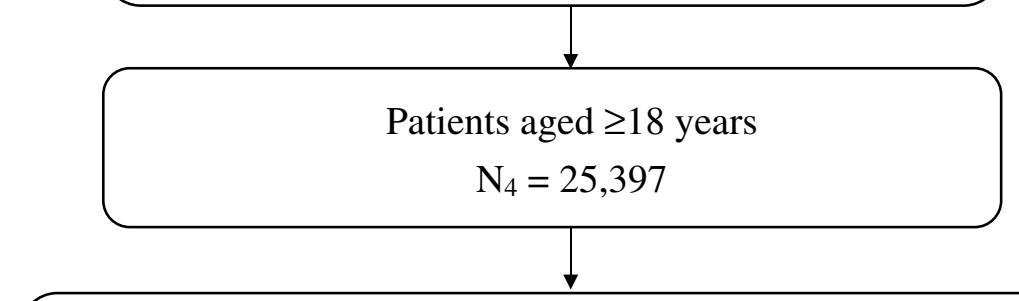

Had continuous insurance enrollment for the 12 months prior and 12 months post the index hospitalization

$$
\mathrm{N}_{5}=23,164
$$

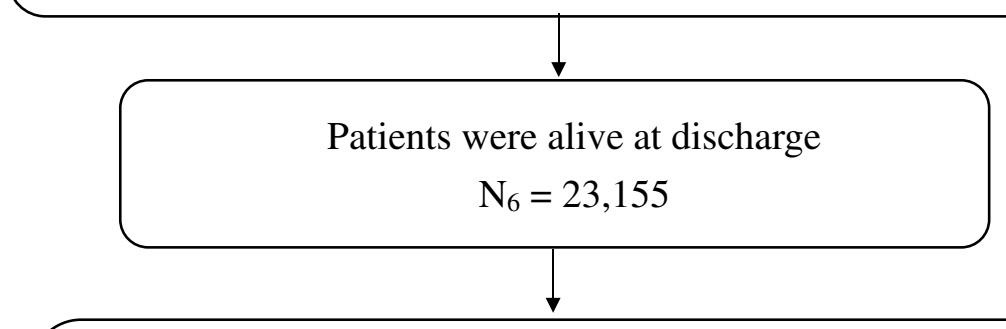

Patients without malignancy and metastatic cancer for the 12 months prior and 12 months post the index

$$
\text { hospitalization }
$$

$$
\mathrm{N}_{7}=22,041
$$

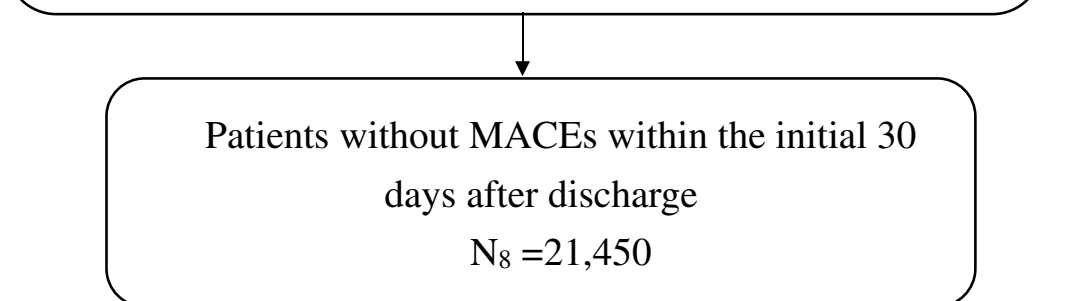

Figure I Flow chart of sample selection. 
Table I Baseline Characteristics

\begin{tabular}{|c|c|c|}
\hline \multirow{2}{*}{$\begin{array}{l}\text { Demographic Characteristics } \\
\text { Age, Mean (SD) }\end{array}$} & \multicolumn{2}{|c|}{$N=21,450$} \\
\hline & 64.6 & 10.7 \\
\hline \multicolumn{3}{|l|}{ Age group, n (\%) } \\
\hline$\leq 54$ & 3,584 & $16.7 \%$ \\
\hline $55-64$ & 7,670 & $35.8 \%$ \\
\hline $65-74$ & 5,731 & $26.7 \%$ \\
\hline$\geq 75$ & 4,465 & $20.8 \%$ \\
\hline Female, n (\%) & 9,861 & $46.0 \%$ \\
\hline Retired, n (\%) & 17,275 & $80.5 \%$ \\
\hline Charlson Comorbidity Index, Mean (SD) & 1.7 & 1.6 \\
\hline \multicolumn{3}{|l|}{ Medical history, n (\%) } \\
\hline Hypertension & 16,090 & $75.0 \%$ \\
\hline Hyperlipidemia & 10,006 & $46.6 \%$ \\
\hline Diabetes mellitus & 8,038 & $37.5 \%$ \\
\hline Prior stroke & 5,586 & $26.0 \%$ \\
\hline Prior angina & 5,388 & $25.1 \%$ \\
\hline Prior myocardial infraction & 3,270 & $15.2 \%$ \\
\hline Prior percutaneous coronary intervention (PCl) & 1,418 & $6.6 \%$ \\
\hline \multicolumn{3}{|l|}{ Prior use, ${ }^{\mathbf{a}} \mathrm{n}(\%)$} \\
\hline Antiplatelet & 11,728 & $54.7 \%$ \\
\hline$\beta$-blockers & 8,801 & $41.0 \%$ \\
\hline Calcium channel blockers & 12,010 & $56.0 \%$ \\
\hline $\begin{array}{l}\text { Antiplatelet agents, angiotensin- } \\
\text { converting enzyme inhibitors/angiotensin } \\
\text { receptor blockers }\end{array}$ & $\mathrm{II}, 446$ & $53.4 \%$ \\
\hline Statins & 9,347 & $43.6 \%$ \\
\hline \multicolumn{3}{|l|}{ Resource utilization and cost } \\
\hline Any hospitalizations, n (\%) & 6,668 & $31.1 \%$ \\
\hline $\begin{array}{l}\text { Number of outpatient visits among all patients, } \\
\text { Mean (SD) }\end{array}$ & 30.5 & 31.7 \\
\hline Total direct medical cost (CNY), Mean (SD) & 12,772 & 18,893 \\
\hline \multicolumn{3}{|l|}{ Index hospitalization } \\
\hline Any PCl, n (\%) & 4,771 & $22.2 \%$ \\
\hline Mean length of stay (days), Mean (SD) & 10.5 & 5.5 \\
\hline
\end{tabular}

Note: ${ }^{\text {a }} \mathrm{Had}$ at least one prescription during the baseline

Table 2 Initiation with Antiplatelet Therapy Within the 30 Days After Discharge

\begin{tabular}{|l|l|l|}
\hline & \multicolumn{2}{|l|}{$\mathbf{N}=\mathbf{2 1}, \mathbf{4 5 0}$} \\
\hline Initiation with antiplatelet therapy, $\mathbf{n}(\%)$ & 15,071 & $70.3 \%$ \\
Aspirin & 10,160 & $47.4 \%$ \\
Clopidogrel & 10,226 & $47.7 \%$ \\
Dual therapy & 5,336 & $24.9 \%$ \\
\hline Initiation without antiplatelet therapy, n (\%) & 6379 & $29.7 \%$ \\
\hline
\end{tabular}

$\mathrm{OR}=1.47[1.26-1.73]$ ) or index hospitalization (adjusted $\mathrm{OR}=22.40 \quad$ [18.63-26.92]) was associated with an increased possibility of using antiplatelet agents within the initial 30 days after discharge. While compared with males, the female patients were less likely to initiate antiplatelet agents (adjusted OR=0.75 [0.70-0.81]).

\section{Persistence With Antiplatelet Agents And Associated Factors}

As presented by the Kaplan-Meier survival curve (Figure 2), the percentage of non-persistent patients increased with time after discharge. During the follow-up period, $85.0 \%$ of patients $(\mathrm{N}=12,809)$ had discontinued the antiplatelet therapy and the average time to discontinuation was 117.4 (119.7) days. Almost half of initial users had discontinuation within 3 months after discharge of the index hospitalization.

The associated factors of persistence were analyzed by using Cox regressions (Table 4). Compared with patients aged $\leq 54$, the likelihood of being persistent was lower for patients aged $\geq 75$ years (adjusted Hazard Ratio [95\% $\mathrm{CI}]=1.08[1.00-1.17])$. And, compared with males, female patients (adjusted HR=1.22 [1.18-1.27]) were more likely to discontinue the antiplatelet treatment. Complications of prior hypertension (adjusted HR=1.10 [1.03-1.17]) and hyperlipidemia (adjusted HR=1.06 [1.01-1.11]) were both risk factors of discontinuation with antiplatelet agents. Baseline utilization of $\beta$-blockers (adjusted HR=1.05 [1.01-1.10]) or CCB (adjusted HR=1.08 [1.03-1.13]) was related with higher risk of discontinuation, while baseline antiplatelet agents utilization (adjusted $\mathrm{HR}=0.78$ [0.74-0.81]) or stain utilization (adjusted $\mathrm{HR}=0.91[0.87-0.96])$ was related with lower risk of discontinuation. In addition, receiving PCI during baseline period (adjusted HR $=0.91$ [0.84-0.97]) or index hospitalization (adjusted HR $=0.51[0.49-0.53]$ ) was associated with significantly lower possibility of discontinuation.

\section{Discussion}

To the best of our knowledge, this is the first observational study which presents the initiation and persistence of antiplatelet treatment in ACS population using real-word data in China. We found that both the initial use and subsequent persistence were poor among ACS patients in Tianjin, China; and the patients who were female were less likely to initiate or persist the treatment pattern, while patients who had no prior antiplatelet agents use or had not received PCI during baseline period or index hospitalization were more likely to initiate and persist that. The findings provide useful information on the precise management of ACS in China. 


\begin{tabular}{|c|c|c|c|c|c|c|c|c|c|c|c|c|c|c|c|c|c|c|c|}
\hline \multirow{4}{*}{ 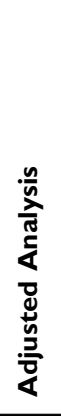 } & 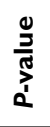 & \multirow{4}{*}{ 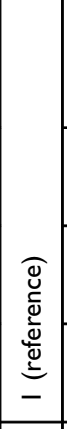 } & $\begin{array}{l}\bar{\delta} \\
\text { iे }\end{array}$ & $\begin{array}{l}\bar{Q} \\
\text { ì }\end{array}$ & $\begin{array}{l}\bar{\delta} \\
\text { i. }\end{array}$ & $\begin{array}{l}\overline{8} \\
\dot{0}\end{array}$ & 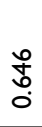 & 气̃ & $\begin{array}{l}\stackrel{+}{0} \\
\text { o } \\
0\end{array}$ & $\begin{array}{l}\text { ơ } \\
\text { o. }\end{array}$ & $\begin{array}{c}\text { के } \\
\text { m. } \\
\text {. }\end{array}$ & 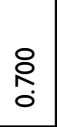 & $\frac{\infty}{0}$ & ষ্. & $\begin{array}{l}\bar{\partial} \\
\stackrel{0}{v}\end{array}$ & $\begin{array}{l}\overline{8} \\
\text { iे }\end{array}$ & s. & 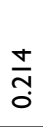 & $\begin{array}{l}\text { مे } \\
\text { مै }\end{array}$ \\
\hline & \multirow[b]{2}{*}{$\begin{array}{c}\bar{u} \\
\text { ㅇํ }\end{array}$} & & ộ & $\stackrel{\grave{i}}{\text { in }}$ & $\stackrel{m}{\underline{\sigma}}$ & $\begin{array}{c}\bar{\infty} \\
0 \\
0\end{array}$ & ơ & $\underline{0}$ & $\underline{\underline{ }}$ & $\stackrel{\infty}{.}$ & $\stackrel{n}{\text { O. }}$ & $\underline{\underline{\theta}}$ & $\stackrel{m}{\stackrel{o}{-}}$ & $\stackrel{\circ}{\stackrel{9}{=}}$ & $\stackrel{n}{=}$ & ઠ్రి & $\underset{\Xi}{\tilde{I}}$ & $\stackrel{m}{\stackrel{0}{-}}$ & $\stackrel{\circ}{\circ}$ \\
\hline & & & $\overline{\underline{m}}$ & $\stackrel{\bar{o}}{-}$ & $\stackrel{g}{t}$ & $\stackrel{R}{\circ}$ & $\begin{array}{c}\hat{\infty} \\
0 \\
0\end{array}$ & $\stackrel{8}{\circ}$ & $\begin{array}{c}\infty \\
\stackrel{\infty}{\infty} \\
0\end{array}$ & హ. & 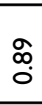 & ㅇ. & $\stackrel{\substack{0 \\
0}}{0}$ & o. & $\stackrel{\text { Iิ }}{=}$ & $\stackrel{\infty}{\stackrel{\sim}{=}}$ & $\stackrel{\text { LO }}{\underline{0}}$ & $\begin{array}{c}\hat{\infty} \\
0 \\
0\end{array}$ & \&. \\
\hline & ڤ̊ & & $\stackrel{f}{=}$ & $\stackrel{\widetilde{\infty}}{-}$ & $\stackrel{R}{R}$ & $\begin{array}{l}n \\
0 \\
0\end{array}$ & o. & $\stackrel{\Delta}{\Delta}$ & $\begin{array}{l}\text { 。 } \\
0\end{array}$ & $\stackrel{8}{\circ}$ & 。̊ & $\stackrel{\infty}{\circ}$ & 今. & $\stackrel{\infty}{=}$ & f́. & $\stackrel{m}{\underline{\alpha}}$ & $\stackrel{m}{=}$ & 。̊ & $\stackrel{\substack{\circ \\
\circ}}{0}$ \\
\hline$\frac{\mathscr{J}}{\frac{\partial}{\pi}}$ & & $\begin{array}{l}\overline{8} \\
\dot{0} \\
\dot{v}\end{array}$ & 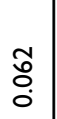 & $\begin{array}{l}\bar{\partial} \\
\dot{0} \\
\dot{v}\end{array}$ & $\frac{\sigma}{0}$ & $\begin{array}{l}\overline{8} \\
\dot{0} \\
\mathrm{v}\end{array}$ & 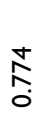 & $\begin{array}{l}\overline{8} \\
\dot{0}\end{array}$ & $\begin{array}{l}\overline{8} \\
\dot{0} \\
\mathrm{v}\end{array}$ & $\begin{array}{l}\bar{\partial} \\
\dot{0} \\
\dot{v}\end{array}$ & $\begin{array}{l}\overline{8} \\
\dot{0} \\
\dot{v}\end{array}$ & 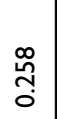 & $\frac{\substack{\infty \\
\infty \\
\infty}}{0}$ & $\begin{array}{l}\overline{8} \\
\text { iे } \\
\mathrm{v}\end{array}$ & $\begin{array}{l}\overline{8} \\
\dot{0} \\
\dot{v}\end{array}$ & $\begin{array}{l}\bar{\delta} \\
\dot{0} \\
\dot{v}\end{array}$ & $\begin{array}{l}\bar{\delta} \\
\dot{0} \\
\dot{v}\end{array}$ & $\begin{array}{l}\overline{8} \\
\dot{\mathrm{v}}\end{array}$ & $\begin{array}{l}\overline{8} \\
\dot{0}\end{array}$ \\
\hline
\end{tabular}

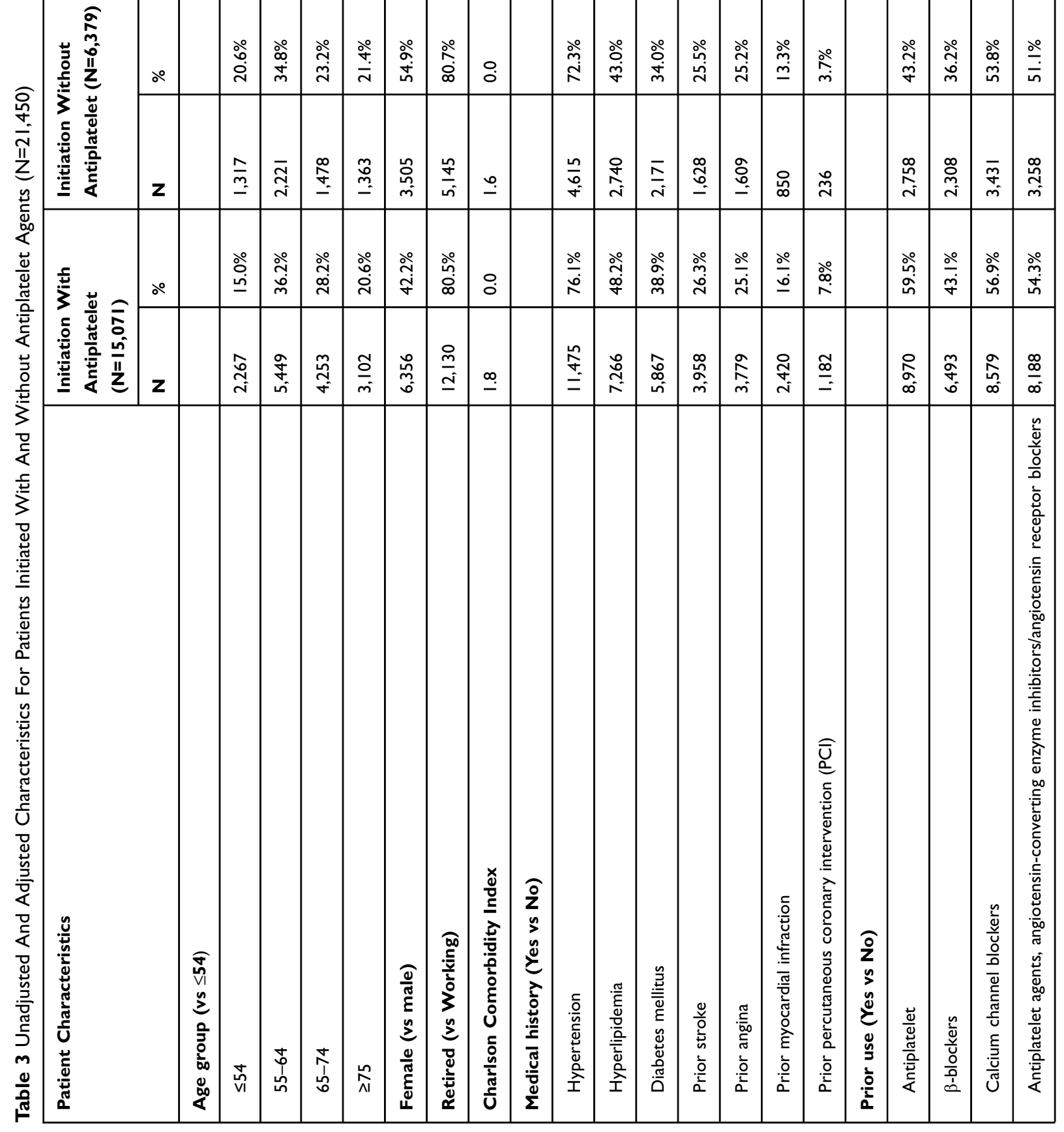




\begin{tabular}{|c|c|c|c|c|c|c|c|}
\hline \multirow{4}{*}{ 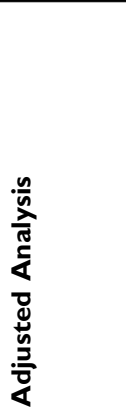 } & 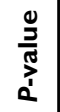 & $\begin{array}{l}\text { fo } \\
\text { o. }\end{array}$ & $\begin{array}{l}\bar{o} \\
\dot{0} \\
\dot{v}\end{array}$ & $\begin{array}{l}\overline{8} \\
\dot{0} \\
\mathrm{v}\end{array}$ & $\begin{array}{l}\text { @ু } \\
\text { o. }\end{array}$ & $\begin{array}{l}\bar{o} \\
\dot{0} \\
\dot{v}\end{array}$ & $\begin{array}{l}\overline{8} \\
\text { ì }\end{array}$ \\
\hline & \multirow[b]{2}{*}{$\begin{array}{l}\bar{u} \\
\text { ㅇํ } \\
\text { 이 }\end{array}$} & $\stackrel{\text { }}{\circ}$ & $\stackrel{\circ}{\circ}$ & $\underline{\underline{\alpha}}$ & $\underset{\sigma}{\sigma}$ & $\begin{array}{l}\alpha \\
\text { dे }\end{array}$ & $\begin{array}{l}\infty \\
\stackrel{0}{0} \\
0\end{array}$ \\
\hline & & 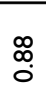 & 華 & $\underset{-}{\not}$ & $\stackrel{\infty}{\circ}$ & $\begin{array}{l}\tilde{3} \\
\infty \\
\underline{\infty}\end{array}$ & å \\
\hline & ๕̊ & o. & : & 호. & $\underset{-}{8}$ & 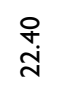 & o. \\
\hline \multicolumn{2}{|l|}{$\frac{\mathscr{D}}{\frac{D}{\pi}}$} & $\begin{array}{l}\bar{\partial} \\
\dot{0} \\
\stackrel{0}{*}\end{array}$ & $\begin{array}{l}\bar{o} \\
\dot{0} \\
\mathrm{v}\end{array}$ & $\begin{array}{l}\bar{\delta} \\
\dot{0} \\
\mathrm{v}\end{array}$ & $\begin{array}{l}\bar{o} \\
\dot{0} \\
\mathrm{v}\end{array}$ & $\begin{array}{l}\bar{o} \\
\dot{0} \\
\mathrm{v}\end{array}$ & $\begin{array}{l}\bar{o} \\
\dot{\mathrm{v}}\end{array}$ \\
\hline \multirow{2}{*}{ 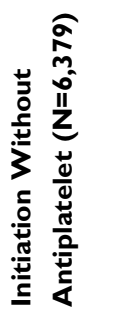 } & ১o & 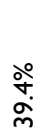 & $\begin{array}{l}\text { ओें } \\
\text { ஸे }\end{array}$ & $\begin{array}{l}\infty \\
\stackrel{\infty}{\sim} \\
\text {. }\end{array}$ & $\begin{array}{l}\overline{\mathrm{i}} \\
\overline{0} \\
\underline{\underline{0}}\end{array}$ & $\stackrel{\circ}{\circ}$ & $\bar{o}$ \\
\hline & $\mathbf{z}$ & $\underset{\substack{n \\
N}}{\stackrel{v}{n}}$ & 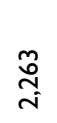 & $\begin{array}{l}\stackrel{n}{\sim} \\
\stackrel{\sim}{\sim}\end{array}$ & $\begin{array}{l}\stackrel{0}{\infty} \\
\stackrel{\infty}{m_{m}} \\
\stackrel{=}{=}\end{array}$ & $\cong$ & $\stackrel{m}{=}$ \\
\hline \multirow{2}{*}{ 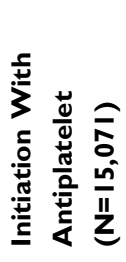 } & ஃீ & 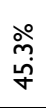 & 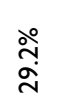 & 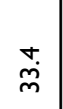 & $\begin{array}{l}+ \\
\stackrel{0}{0} \\
\alpha \\
\alpha \\
\sigma\end{array}$ & $\begin{array}{l}\stackrel{\circ}{\circ} \\
\stackrel{m}{0} \\
\end{array}$ & $\stackrel{\circ}{\circ}$ \\
\hline & $\mathbf{z}$ & 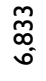 & 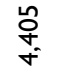 & $\begin{array}{l}\stackrel{L}{\dot{m}} \\
\text {. }\end{array}$ & $\begin{array}{c}\stackrel{0}{m} \\
\text { m}\end{array}$ & \begin{tabular}{l} 
我 \\
\multirow{\sigma}{*}{}
\end{tabular} & ํㅗ \\
\hline 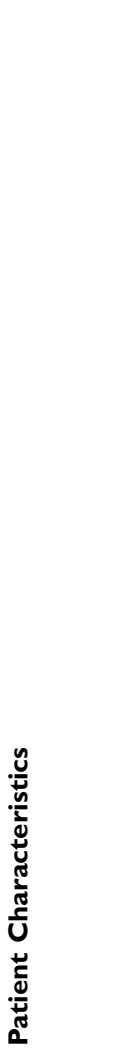 & & 总 & 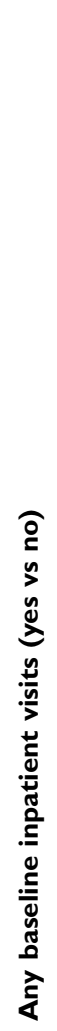 & 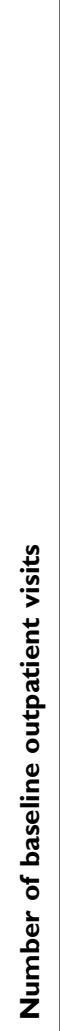 & 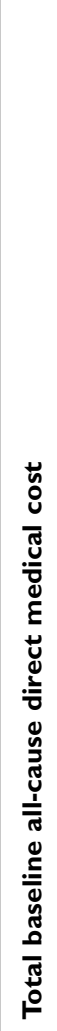 & 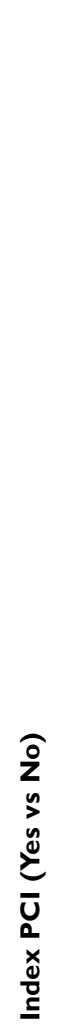 & 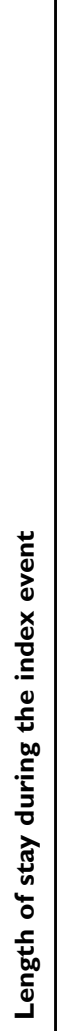 \\
\hline
\end{tabular}

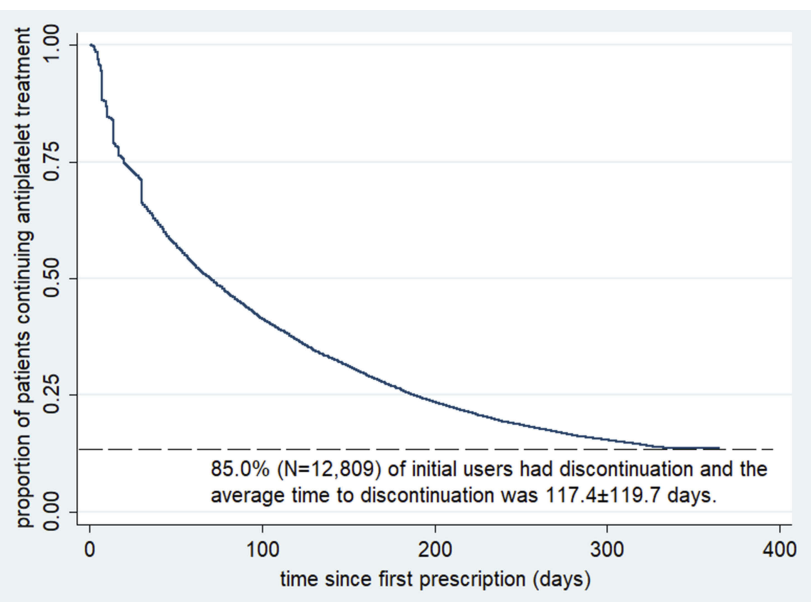

Figure 2 Persistence with antiplatelet agents during the follow-up period.

Compared with the results of previous studies from different countries, the proportion of initiation of antiplatelet agents was relatively lower (70\%) within the 30 days after discharge and the proportion of discontinuation was much higher (85\%) during the 12 months after discharge in the present study. In the previous studies, it was reported that more than $90 \%$ of ACS patients possessed prescription of antiplatelet agents at discharge. ${ }^{18,22,28} \mathrm{As}$ for persistence, the previous retrospective studies using a large observational database from different countries in Europe indicated that $50-73 \%$ of patients had discontinuation of antiplatelet agents with gaps $>30$ days during 1year follow-up in patients after an ACS. ${ }^{18,29}$ There are many possible reasons for the poorer initiation and persistence in this study. First, physicians may be unfamiliar with treatment recommendations and/or unaware of the importance of preventive treatment. According to the previous studies, "not indicated" by the physician was recorded as the most common reason for not prescribing medications, which means that the medication was not prescribed because of a perceived lack of indication rather than the presence of a contraindication or intolerance. ${ }^{21,30}$ Second, heavy economic burden of ACS is a potential barrier. Despite the universal health insurance coverage in China, patients still need to pay a proportion of total medical costs (ie, out-of-pocket cost). It reported that the mean annual ACS-related direct medical costs was 8,531 CNY among the Chinese ACS patients, while the per capita disposable income was only $18,311 \mathrm{CNY} .^{31}$

Compared with males, female patients were less likely to initiate with antiplatelet treatment but more likely to discontinue. The results were consistent with the previous 


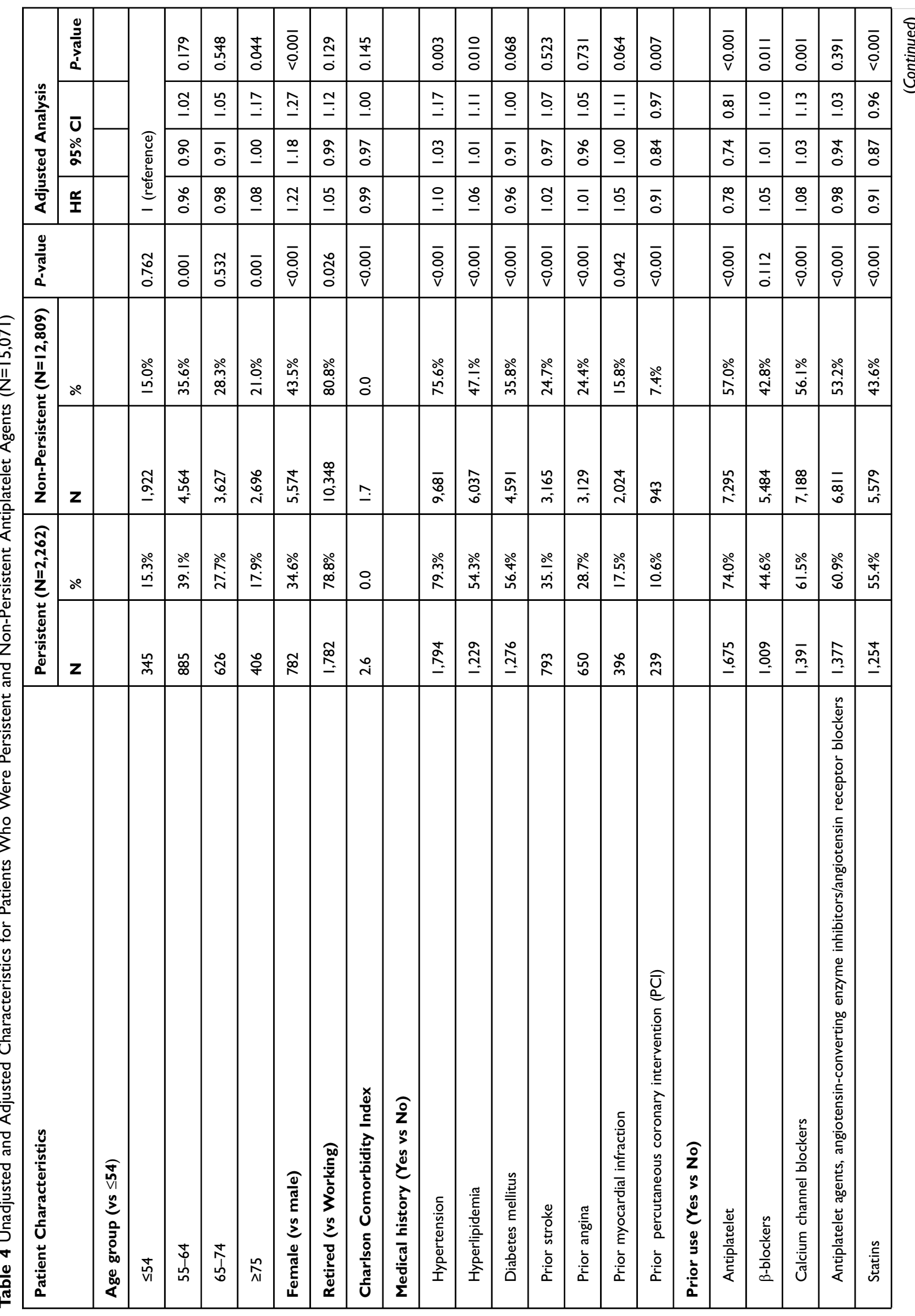




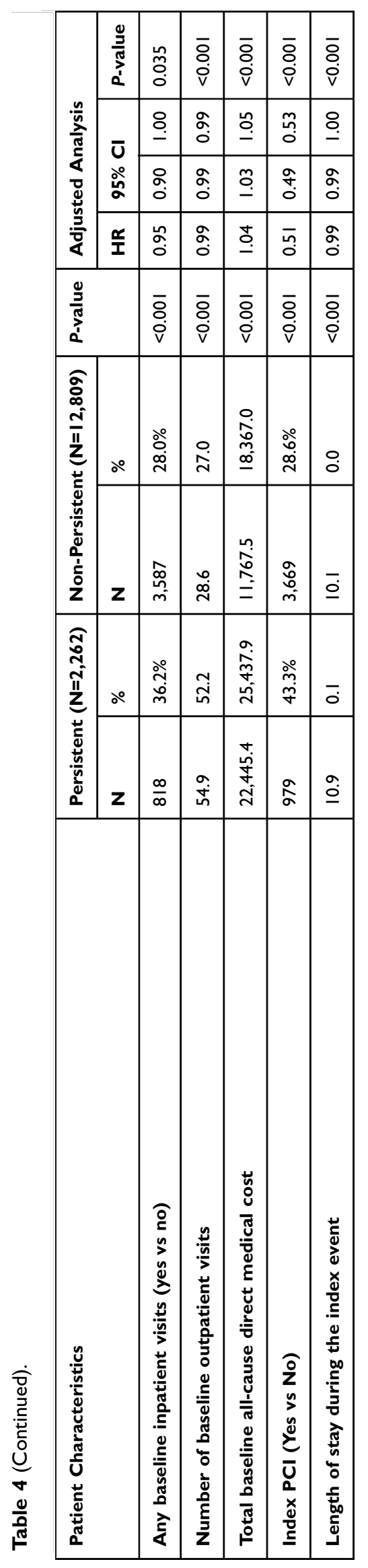

studies in Finland and Denmark. ${ }^{18,32}$ The possible reason for this finding is that females have a higher risk of bleeding and refractory ischemia than males. ${ }^{32,33}$ Despite the guidelines recommending clopidogrel treatment to all patients regardless of gender, treatment with antiplatelets should be considered for every individual fulfilling indications. $^{32,34}$ The results suggested that physicians should pay more attention to the antiplatelet management of females.

The study found that patients who had any prescription of antiplatelet agents during the baseline period were more likely to initiate and persist the follow-up antiplatelet treatment. A Finnish study also reported that the patients who used antiplatelet agents during the baseline period were 3.7-times more likely of initiating antiplatelet after ACS and half the risk of discontinuing than those who had no records of antiplatelet utilization. ${ }^{18}$ The patients who used antiplatelet agents prior to index hospitalization indicated that they did not have any contraindication, allergy history, or adverse drug reaction. They may have already formed a good habit for antiplatelet agents utilization, which is a possible reason for higher compliance with the guidelines.

The results from this study suggested receiving PCI during the index hospitalization was positively related to both initiation and persistence, which was consistent with previous studies that reported patients who received PCI during index hospitalization had a nearly 20 -fold possibility of initiation with antiplatelet agents and faced lower risk of non-persistence than those who did not receive PCI. ${ }^{18,23}$ The results may be related to the guidelines in China. The most updated international guidelines for ACS during the study period recommend that all the patients with ACS receiving PCI or not should use the antiplatelet treatment of aspirin and clopidogrel from the discharge of the first ACS up to 12 months. ${ }^{1,15}$ Despite the Chinese guidelines recommending that all patients with STE-MI should use the aspirin and clopidogrel whether with or without PCI, the clopidogrel only be recommend for the NSTE-MI/UA patients with PCI., ${ }^{9,16}$ In clinical practice, physicians usually highly recommend the DAPT for the ACS patients with PCI in China. This may explain why the patients with PCI tend to have better initiation and persistence in the present study.

The present study does have some limitations. First, aspirin could be obtained OTC and ticagrelor was not reimbursed by UEBMI (during the study period) and this may have caused the underestimation. However, because 
of the high price without reimbursement by insurance, the market share of other $\mathrm{P} 2 \mathrm{Y}_{12}$ inhibitors was very limited during the study period in China, which resulted in minimal influence on the study. Secondarily, the claim database has no detailed information of socioeconomics, living habits, and disease severity, thus the analyses exploring the factors associated were not able to control these unmeasured factors, which may be associated with initiation and persistence. Furthermore, due to the limitation of the database, estimates of both initiation and persistence in this study were based on patterns of drugs dispensed, but not necessarily consumed. Additionally, the generalizability is limited due to data from an insured population under employment and those who had retired in Tianjin. Although there are some limitations, this study provides real-world evidence that helps discover problems and promote management of secondary prevention for ACS.

\section{Conclusion}

The initiation of antiplatelet therapy is unsatisfactory among the ACS patients in Tianjin, China, and the persistence to antiplatelet is poor among the initial users. It represents suboptimal treatment practices as well as opportunities for improvement. The study found that patients who are female, have no prior antiplatelet agent utilization, and do not receive PCI during the baseline period or index hospitalization are the population at risk of non-initiation and non-persistence with antiplatelet treatment, and who need more attention from the physicians.

\section{Disclosure}

This study was funded by Sanofi China. The authors have indicated that they have no other conflict of interest with regard to the content of this article.

\section{References}

1. Amsterdam EA, Wenger NK, Brindis RG, et al. 2014 AHA/ACC guideline for the management of patients with Non-ST-elevation acute coronary syndromes: a report of the American College of Cardiology/American Heart Association Task Force on Practice Guidelines. J Am Coll Cardiol. 2014;64(24):e139-e228.

2. Ke YN, Chen JL. Guideline for the management of patients with unstable angina or non ST-elevation acute coronary syndromes [in Chinese]. Chin J Cardiol. 2007;35(4):295-304.

3. Smith JN, Negrelli JM, Manek MB, Hawes EM, Viera AJ. Diagnosis and management of acute coronary syndrome: an evidence-based update. J Am Board Fam Med. 2015;28(2):283-293.

4. Chiang FT, Shyu KG, Wu CJ, et al. Predictors of 1-year outcomes in the Taiwan acute coronary syndrome full spectrum registry. $J$ Formos Med Assoc. 2014;113(11):794-802.
5. Axelsen PH, Bajzer Z, Prendergast FG. Prospective multi-center study of female patients with ST-elevation myocardial infarction in Liaoning province, China. Chin Med J. 2012;125(11):1915-1919.

6. Rasmussen JN, Chong A, Alter DA. Relationship between adherence to evidence-based pharmacotherapy and long-term mortality after acute myocardial infarction. JAMA. 2007;297(2):177.

7. Hamood H, Hamood R, Green MS, Almog R. Effect of adherence to evidence-based therapy after acute myocardial infarction on all-cause mortality. Pharmacoepidemiol Drug Saf. 2015;24(10):1093.

8. Austin PC, Mamdani MM, Juurlink DN, Alter DA, Tu JV. Missed opportunities in the secondary prevention of myocardial infarction: an assessment of the effects of statin underprescribing on mortality. Am Heart J. 2006;151(5):969-975.

9. Chinese Society of Cardiology, Editorial Board of Chinese Journal of Cardiology. Guideline for the management of patients with non STelevation acute coronary syndromes [in Chinese]. Chin J Cardiol. 2012;40(5):353-367.

10. Yan AT, Yan RT, Tan M, et al. Optimal medical therapy at discharge in patients with acute coronary syndromes: temporal changes, characteristics, and 1-year outcome. Am Heart J. 2007;154(6):1108-1115.

11. Amsterdam EA, Wenger NK, Brindis RG, et al. 2014 AHA/ACC guideline for the management of patients with non-ST-elevation acute coronary syndromes: executive summary A Report of the American College of Cardiology/American Heart Association Task Force on Practice Guidelines. Duke University Press, 2014: 121-123.

12. Crawshaw J, Auyeung V, Norton S, et al. Identifying psychosocial predictors of medication non-adherence following acute coronary syndrome: a systematic review and meta-analysis. $J$ Psychosom Res. 2016;90:10.

13. Green A, Pottegård A, Broe A, et al. Initiation and persistence with dual antiplatelet therapy after acute myocardial infarction: a Danish nationwide population-based cohort study. BMJ Open. 2016;6(5):e010880.

14. Friedman H, Mollon P, Lian J, et al. Clinical outcomes, health resource use, and cost in patients with early versus late dual or triple anti-platelet treatment for acute coronary syndrome. Am J Cardiovasc Drugs. 2013;13(4):273.

15. Damman P, Hof AWVT, Berg JMT, et al. 2015 ESC guidelines for the management of acute coronary syndromes in patients presenting without persistent ST-segment elevation: comments from the Dutch ACS working group. Neth Heart J. 2017;25(3):181-185.

16. Chinese Society of Cardiology, Editorial Board of Chinese Journal of Cardiology. Guidelines for the management of acute myocardial infarction in patients presenting with ST segment elevation [in Chinese]. Chin J Cardiol. 2015;43(5):380-393.

17. Steinhubl SR, Berger PB, Mann JT 3rd, et al. Early and sustained dual oral antiplatelet therapy following percutaneous coronary intervention: a randomized controlled trial. JAMA. 2002;288(19):2411-2420.

18. Prami T, Khanfir H, Deleskog A, et al. Clinical factors associated with initiation of and persistence with ADP receptor-inhibiting oral antiplatelet treatment after acute coronary syndrome: a nationwide cohort study from Finland. BMJ Open. 2016;6(11):e012604.

19. Osterberg L, Blaschke T. Adherence to medication. $N$ Engl J Med. 2005;353(18):1972-1974. doi:10.1056/NEJMra050100

20. Silcock J, Standage C. Exploring patient adherence to cardiovascular medicines. Br J Nurs. 2013;2(5):223-228.

21. Bi Y, Gao R, Patel A, et al. Evidence-based medication use among Chinese patients with acute coronary syndromes at the time of hospital discharge and 1 year after hospitalization: results from the Clinical Pathways for Acute Coronary Syndromes in China (CPACS) study. Am Heart J. 2009;157(3):509-516.e1.

22. Redfern J, Hyun K, Chew DP, et al. Prescription of secondary prevention medications, lifestyle advice, and referral to rehabilitation among acute coronary syndrome inpatients: results from a large prospective audit in Australia and New Zealand. Heart. 2014;100 (16):1281-1288. 
23. Boggon R, Staa TPV, Timmis A, et al. Clopidogrel discontinuation after acute coronary syndromes: frequency, predictors and associations with death and myocardial infarction-a hospital registry-primary care linked cohort (MINAP-GPRD). Eur Heart J. 2011;32 (19):2376-2386.

24. Pinnarelli L, Mayer F, Bauleo L, et al. Adherence to antiplatelet therapy after percutaneous coronary intervention: a population study in a region of Italy. J Cardiovasc Med. 2015;16:3.

25. Sanfélixgimeno G, Peiró S, Ferreros I, et al. Adherence to evidencebased therapies after acute coronary syndrome: a retrospective population-based cohort study linking hospital, outpatient, and pharmacy health information systems in Valencia, Spain. J Manag Care Pharm. 2013;19(3):247.

26. Latry P, Martinlatry K, Lafitte M, et al. Dual antiplatelet therapy after myocardial infarction and percutaneous coronary intervention: analysis of patient adherence using a French health insurance reimbursement database. Eurointervention. 2012;7(12):1413.

27. Tianjin Statistics Bureau. Statistical communique of Tianjin on the 2015 national economic and social development. 2016. Available from: http:// stats.tj.gov.cn/Item/25858.aspx. Accessed November 5, 2019.

28. Kerr AJ, Turaga M, Grey $\mathrm{C}$, et al. Initiation and maintenance of statins and aspirin after acute coronary syndromes (ANZACS-QI 11). J Prim Health Care. 2016;8(3):238-249.
29. Claeys MJ, Beauloye C, Pourbaix S, et al. Real world insights on the initiation and treatment duration of oral antiplatelets in acute coronary syndromes: a retrospective cohort study. Eur Heart J Cardiovasc Pharmacother. 2017;3(4):189-197.

30. He X, Wang Y, Cong H, Lu C, Wu J. Impact of optimal medical therapy at discharge on one-year direct medical costs in patients with acute coronary syndromes: a retrospective, observational database analysis in China. Clin Ther. 2019;41(3):456-465.e2. doi:10.1016/j. clinthera.2019.01.005

31. Wang Y, Cong H, Lu C, et al. Healthcare resource utilization and cost of patients with acute coronary syndromes in Tianjin, China. Value Health. 2017;20(9):A502.

32. Sørensen R, Gislason GH, Fosbøl EL, et al. Initiation and persistence with clopidogrel treatment after acute myocardial infarction: a nationwide study. Br J Clin Pharmacol. 2008;66(6):875

33. Tangri N, Ferguson TW, Whitlock RH, et al. Long term health outcomes in patients with a history of myocardial infarction: a population based cohort study. PLoS One. 2017;12(7):e0180010.

34. Anand SS, Xie CC, Mehta S, et al. Differences in the management and prognosis of women and men who suffer from acute coronary syndromes. J Am Coll Cardiol. 2005;46(10):1845-1851. doi:10.1016/ j.jacc.2005.05.091.
Patient Preference and Adherence

\section{Publish your work in this journal}

Patient Preference and Adherence is an international, peer-reviewed, open access journal that focusing on the growing importance of patient preference and adherence throughout the therapeutic continuum. Patient satisfaction, acceptability, quality of life, compliance, persistence and their role in developing new therapeutic modalities and compounds to optimize clinical outcomes for existing disease

\section{Dovepress}

states are major areas of interest for the journal. This journal has been accepted for indexing on PubMed Central. The manuscript management system is completely online and includes a very quick and fair peer-review system, which is all easy to use. Visit http:// www.dovepress.com/testimonials.php to read real quotes from published authors. 